Authors

B. Viticchié, M. Vantaggiato, F. Berrilli, D. del Moro, V. Penza, E. Pietropaolo, and M. Rast 


\title{
Modeling the solar irradiance background via numerical simulation
}

\author{
B. Viticchié • M. Vantaggiato $\cdot$ F. Berrilli $\cdot$ D. del Moro • \\ V. Penza $\cdot$ E. Pietropaolo $\cdot$ M. Rast
}

Received: 28 October 2009 / Accepted: 26 January 2010 / Published online: 11 February 2010

(c) Springer Science+Business Media B.V. 2010

\begin{abstract}
Various small scale photospheric processes are responsible for spatial and temporal variations of solar emergent intensity. The contribution to total irradiance fluctuations of such small scale features is the solar irradiance background. Here we examine the statistical properties of irradiance background computed via a $n$-body numerical scheme mimicking photospheric space-time correlations and calibrated by means of IBIS/DST spectro-polarimetric data. Such computed properties are compared with experimental results derived from the analysis of a VIRGO/SPM data. A future application of the model here presented could be the interpretation of stellar irradiance power spectra observed by new missions such as Kepler.
\end{abstract}

Keywords Solar irradiance background

B. Viticchié $(\bowtie)$

European Space Agency (ESTEC), PO Box 299, 2200 AG,

Noordwijk, The Netherlands

e-mail: Bartolomeo.Viticchie@esa.int.it

B. Viticchié · M. Vantaggiato · F. Berrilli · D. del Moro · V. Penza Dipartimento di Fisica, Universitá degli Studi di Roma "Tor Vergata", Via della Ricerca Scientifica 1, 00133 Rome, Italy

E. Pietropaolo

Dipartimento di Fisica, Universitá degli Studi di L’Aquila, Via Vetoio Localitá Coppito, 67100 L'Aquila, Italy

M. Rast

Laboratory for Atmospheric and Space Physics, Department of Astrophysical and Planetary Science, University of Colorado, Boulder, CO 80309, USA

\section{Introduction}

The fast and erratic variations, in time and space, of small scale solar surface features $\left(\sim 10^{3} \mathrm{~km}\right)$ produce an integrated high frequency noise signal called Solar Irradiance Background (Rabello Soares et al. 1997). Such a high frequency signal is the response to rapid change in physical properties associated to surface magnetic and convective features.

Space-borne measurements of solar irradiance performed since 1978 show that the irradiance background varies on all accessible time scales (Willson and Hudson 1988; Fröhlich 1994; Fröhlich and Lean 1998). However, the physical scenario behind this variation remains a subject of debate.

For short time scales (i.e., between few days and few minutes), the source of variability is still not well understood. Many models have been proposed to reproduce such rapid variations in solar surface irradiance by means of granulation, mesogranulation and supergranulation (Andersen et al. 1994; Rabello Soares et al. 1997) or granulation and magnetic fields (Solanki et al. 2003).

Here we present a simple irradiance model based on a simulation of photospheric downflow dynamics in which the velocity field is characterized by space-time correlations in agreement with the ones observed on the solar surface. In this paper we explore the reliability of our model to mimic stellar surface space and time correlations possibly responsible for the different power spectrum indices of the intensity background. The modeled stellar surface space-time correlations are analogous to the observed solar granulation-mesogranulation (see Viticchié et al. 2006; Berrilli et al. 2008). The temporal evolution of photospheric downflow plumes produced by the model is here exploited to mimic the variation of the intensity background due to the appearance/disappearance of downflows on the solar photosphere. The intensity contribution of each downflow plume 
is calibrated through spectroscopic and broad-band observations.

\section{Dataset and calibration}

To obtain the relationship between the velocity field and the intensity that will be implemented in the irradiance model, we used observations performed at the NSO/Dunn Solar Telescope in November 21, 2006 from 16:24 UT to 17:17 UT. These consist of a time sequence of a 2.3 pm-step sampling of the FeI $630.15 \mathrm{~nm}$ photospheric line plus simultaneous broad-band $(633.32 \pm 5 \mathrm{~nm})$ counterpart. The whole sequence is of 36 images with a time cadence of $90 \mathrm{sec}$. The target of the observation was a 39.1" $\times 39.1^{\prime \prime}$ quiet Sun region at disk center. The velocity along the line-of-sight for each point of the field-of-view has been calculated via Fourier transform.

We derived a dependence between broad-band intensity normalized to the average intensity over the whole time sequence $\left(I_{\mathrm{BB}}\right)$ and the velocity along the line-of-sight $\left(v_{\mathrm{los}}\right)$. To do this we considered average broad-band intensity values calculated over $65 \mathrm{~m} \mathrm{~s}^{-1}$ velocity bins. A 3 rd order polynomial fit has been performed to obtain an analytic relation between $I_{\mathrm{BB}}$ and $v_{\text {los }}$ (see Fig. 1).

\section{Irradiance model}

\subsection{Dynamical model of photospheric velocity field}

The model simulates the dynamics and evolution of photospheric downflow plumes on the solar surface as resulting from an advection profile modeled as in Rast (1998). The movement of each plume at each simulation step is defined by the interaction with the other plumes in the computational domain. This model, originally proposed by Rast
(2003) to investigate the origin of solar surface convective scales, spontaneously creates stable downflows over mesogranular pattern from the sole interaction taking place over granular scales (for a detailed description of the model, see Viticchié et al. 2006; Berrilli et al. 2008).

\subsection{Irradiance model}

In the preliminary version of the irradiance model, the upflow contribution is not taken into account, i.e., the power spectrum is defined by the intensity variation due to the sole appearance/disappearance of downflows on the solar surface. This choice is motivated by the fact that the dynamical model described in Sect. 3.1 only mimics the evolution of downflows on the solar photosphere. This means that, referring to the polynomial fit reported in Fig. 1, we take into account only the relation for positive values of the line-ofsight velocity. The simple idea to model the time variation of the irradiance is to rescale the downflow velocity range retrieved by the dynamical model to the one obtained from observations. More in detail, we associated the velocity measured in the strongest observed downflow to the velocity of the strongest downflow in each simulation. ${ }^{1}$ In this way, we can easily get a relation between the downflow velocities from the simulation and the observed broad-band intensity values. By integrating the contribution of the intensities related to all the downflows we get the temporal evolution of irradiance from the model.

The model simulates the irradiance emerging from a small portion of solar surface (about $30 \times 30 \mathrm{Mm}^{2}$ ) that is considered to be representative of the properties of the solar irradiance integrated over the whole disk.

\footnotetext{
${ }^{1}$ This association can be done since the values of the velocity from the model are represented with arbitrary units.
}

Fig. 1 Broad-band intensity vs. velocity along the line-of-sight for the whole dataset (yellow scatter plot). Triangles represent average broad-band intensity values calculated over $65 \mathrm{~m} \mathrm{~s}^{-1}$ velocity bins; error bars are calculated as $10 \times \sigma / \sqrt{N}$, where $N$ is the number of counts in each bin. The red line represents the 3 rd order polynomial fit

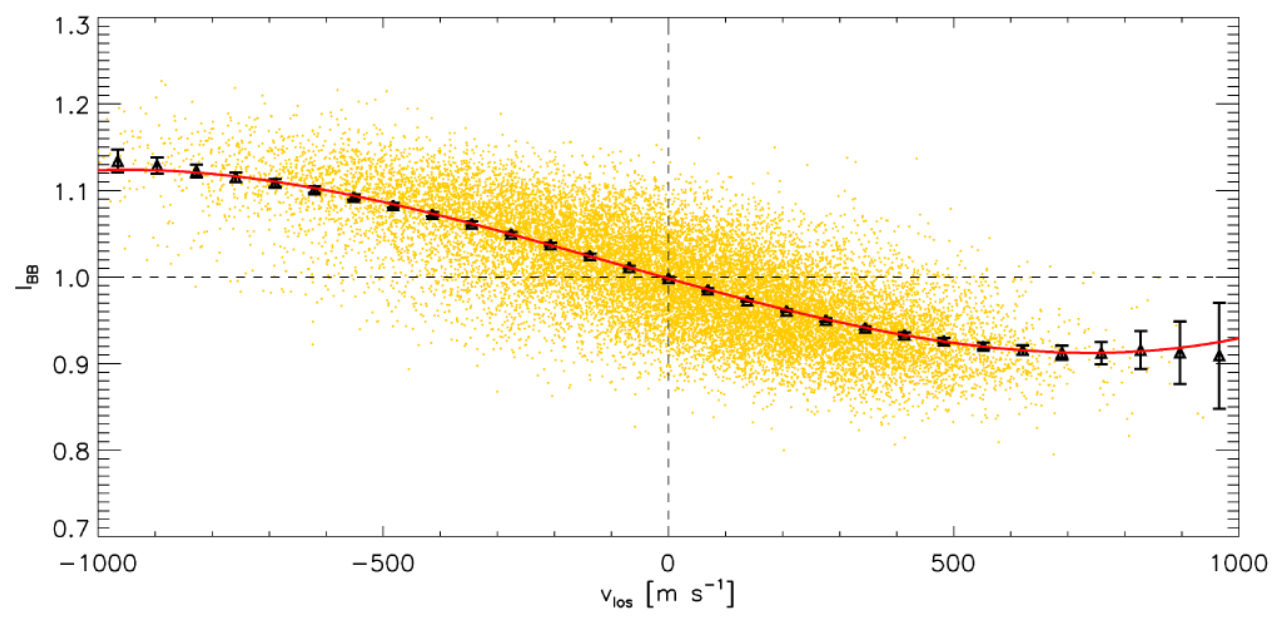




\section{Preliminary results}

Four simulations have been performed, each representing an equivalent of about 2000 min of photospheric evolution with a temporal resolution $\lesssim 1 \mathrm{~min}$. The total time interval of the simulations is set to be larger than the mesogranular time scale (i.e., the largest time scale produced by the model). The four simulations differ only in the initial spatial distribution of the plumes that is set randomly. The variation of the intensity with time derived from the four simulations (Fig. 2, left panel) allows to calculate four simulated power spectra in the frequency range $10^{2}-10^{4} \mu \mathrm{Hz}$ (Fig. 2, right panel); the results are shown in comparison with the power spectrum derived from VIRGO/SPM ${ }^{2}$ data with a temporal cadence of one minute.

The simple irradiance model here described is able to reproduce the right power spectrum for frequencies between $10^{2}-0.5 \times 10^{3} \mu \mathrm{Hz}$. In the range $0.5 \times 10^{3}-10^{4} \mu \mathrm{Hz}, \mathrm{a}$ strong deviation with respect to the observational spectrum is found, indicating a too high power associated to highfrequencies.

We have to remember that, at the present stage, we are not taking into account any information related to the spatial extension of the downflows on the $30 \times 30 \mathrm{Mm}^{2}$ domain; i.e., downflows chains extending over typical scales of few arcseconds should be identified as a single intergranular lane, not as many point-like downflows. From this follows that

${ }^{2}$ See the VIRGO/SPM web page: http://www.ias.u-psud.fr/virgo.

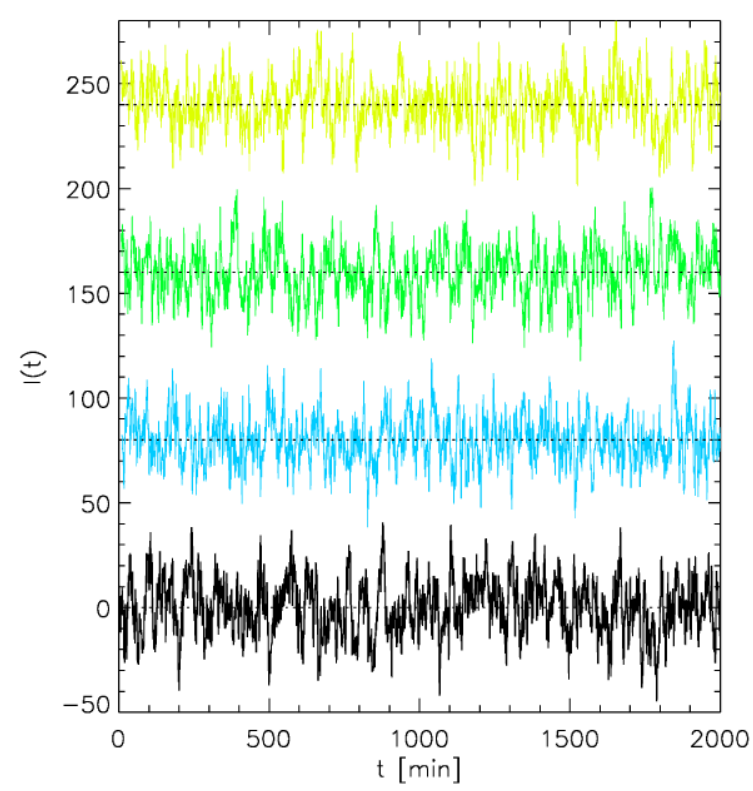

Fig. 2 Left panel: temporal variation of the intensity as derived by the four simulations (here reported in arbitrary units). Right panel: Power spectrum derived from the four curves in the left panel; the the effect of a large number of highly-variable downflows produces the obtained excess of power at high frequencies.

\section{Conclusions}

The knowledge of the solar irradiance background is of great importance for solar and stellar physics. Here we present a model for the description of the solar irradiance background based on a numerical simulation of the photospheric velocity field (Rast 2003).

The present implementation of the irradiance model presented in Sect. 3 allows a good description of the portion of the irradiance spectrum in the $10^{2}-0.5 \times 10^{3} \mu \mathrm{Hz}$ frequency range. A new implementation of the code, to take into account the effect of the spatial coherence of the downflows into spatially extended intergranular lanes should allow a much better description of the spectrum also in the range $0.5 \times 10^{3}-10^{4} \mu \mathrm{Hz}$, where the model retrieves an excess of power; we are currently working on this aspect.

A further aspect to take into account is that the $n$-body simulation here used has been tuned via free parameters to reproduce the solar photosphere space-time correlations (Viticchié et al. 2006). A further step in our investigation will be to check whether the modification of the simulated space-time scales affect the irradiance background spectrum. If so, the model could help us to perform a classification of the stellar surface properties through the analysis of the irradiance power spectrum. The NASA Kepler mission ${ }^{3}$

${ }^{3}$ See the Kepler mission web page: http://kepler.nasa.gov.

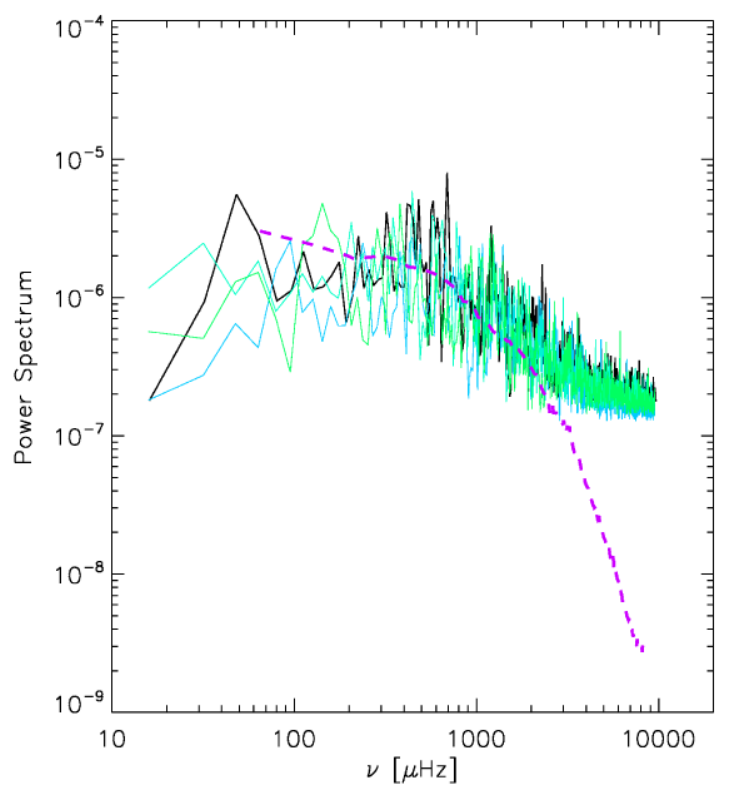

different colors adopted refer to the ones used in the left panel. The VIRGO/SPM power spectrum is also reported in the plot (pink dashed line) 
will observe all stars brighter than $V \simeq 11.5$ at a cadence of 1 minute over a period of 1-3 months in order to analyze p-mode oscillations. These observations will be optimal to compute intensity background power spectrum of the observed stars and derive space and time correlations from our model.

Acknowledgements The authors are grateful to Thierry Appourchaux for providing VIRGO/SPM data.

\section{References}

Andersen, B.N., Leifsen, T.E., Toutain, T.: Sol. Phys. 152, 247 (1994)
Berrilli, F., Del Moro, D., Viticchiè, B.: Astron. Astrophys. 489, 763 (2008)

Fröhlich, C.: In: Pap, J.M., et al. (eds.) IAU Colloq. 143, The Sun as a Variable Star: Solar and Stellar Irradiance Variations, p. 28. Cambridge Univ. Press, Cambridge (1994)

Fröhlich, C., Lean, J.: Geophys. Res. Lett. 25, 4377 (1998)

Rabello Soares, M.C., Roca, Cortes T., Jimenez, A., Anderson, B.N., Appourchaux, T.: Astron. Astrophys. 328, 970 (1997)

Rast, M.P.: J. Fluid Mech. 369, 125 (1998)

Rast, M.P.: Astrophys. J. 597, 1200 (2003)

Solanki, S.K., Seleznyov, A.D., Krivova, N.A.: Eur. Space Agency Spec. Publ. 535, 285 (2003)

Viticchié, B., Del Moro, D., Berrilli, F.: Astrophys. J. 652, 1734 (2006)

Willson, R.C., Hudson, H.S.: Nature 332, 810 (1988) 